\title{
La formación de educadores infantiles, un reto de innovación educativa en la educación superior
}

\section{Childhood educator training, a challenge to educative innovation in higher education}

\author{
Zaily del Pilar García Gutiérrez ${ }^{1}$, Inmaculada Aznar Diaz ${ }^{2}$ \\ ${ }^{1}$ Corporación Universitaria Minuto de Dios, Colombia (zaily.garcia@gmail.com) \\ ${ }^{2}$ Departamento Didáctica y Organización Escolar, Universidad de Granada, España (iaznar@ugr.es)
}

\section{RESUMEN:}

Este artículo surge del proyecto titulado "Análisis de la Formación de Pedagogos Infantiles en la Corporación Universitaria Minuto de Dios Bogotá Colombia", con el propósito presentar los resultados respecto a la pertinencia de la propuesta formativa de pedagogos infantiles de UNIMINUTO, de acuerdo a las necesidades de formación las infancias, como punto de partida para establecer acciones de mejora y transformación del programa, direccionados a generar un aporte de innovación en la educación superior. La metodología, de tipo mixto, se lleva a cabo mediante: la encuesta, el grupo focal y entrevistas, aplicadas a estudiantes, graduados, docentes y directivas respectivamente.

Finalmente, los resultados, donde se expresa una valoración favorable respecto a la pertinencia de la propuesta de formación sobre las cambiantes necesidades educativas de las infancias, en tanto son reconocidas como una representación social completamente determinada por el momento historio y realidad social actual. Sin embargo, se identifica un gran reto de mejora, relacionado con la diversificación de los escenarios de práctica formativa y cantidad de créditos destinados para dicho propósito. Necesidad de transformación que surge como respuesta ante las exigencias de innovación en educación superior.
PALABRAS
CLAVE:
INNOVACIÓN
EDUCATIVA,
EDUCACIÓN
SUPERIOR,
PEDAGOGIA INFANTIL, COMPETENCIAS
FORMATIVAS.

\begin{abstract}
:
This article emerges from the project called "Analysis of the childhood educators training at CorporaciónUniversitariaMinuto de Dios. Bogotá Colombia", with the purpose of presenting the results related to the appropriateness of the formative proposal of childhood educators at UNIMINUTO, according to the childhood's training needs, as a starting point to stablish the improvement actions in higher education. The mixed methodology is carried out through surveys, focus group and interviews applied to students, graduates, professors and administrators respectively.

Finally, the resultsshow a positive assessment related to the relevance of the training proposal about the childhood's changeable educative needs, while they are recognized as a social representation, completely determined by the historical moment and current social reality. However, it is identified a big improvement challenge, associated to the diversification of formative practice scenarios and the quantity of academic credits destined for the aforementioned purpose. That transformation need emerges as a response to the innovation demands in higher education.
\end{abstract}

KEYWORDS: EDUCATIONAL INNOVATION, HIGHER EDUCATION, CHILDREN PEDAGOGY, TRAINING COMPETENCES 


\section{INTRODUCCIÓN}

En un mundo en el que la globalización, asociada a la revolución tecnológica, producción de conocimiento, circulación de la información y masificación de la comunicación (Castells, 1999; Mejía, 2006) la infancia y su formación se configuran en un eje fundamental para la sociedad del presente y el futuro, dado el rol que tienen en la humanidad.

La infancia, como una representación social (Casado, 2001; Jodelet, 2007; Moscovici, 1985) hoy más que nunca, se caracteriza por ser plural, singular y diversa, con la capacidad infinita de transformarse constantemente (Alzate, 2002, 2003; Casas, 1998, 2006; Diker, 2009).

En este orden de ideas la Educación Infantil, se presenta como acto complejo y sistémico (Diker, 2009; Gervilla, 2002, 2006; Peralta, 2003; Zabalza, 1996, 2008) que debe estar en capacidad de hacer de la educación una experiencia vital, multidimensional y singular, diseñada para responder a la pluralidad de las infancias y, así mismo, a las necesidades de la realidad actual del conocimiento y la tecnología (Amar, Abello y Tirado, 2014; Arnaiz, 2003; Trujillo y Marín 2010).

En coherencia con lo anterior, la formación de pedagogos infantiles, surge como un reto de innovación educativa para las instituciones de educación superior, escenarios académicos convocados a responder ante las cambiantes exigencias de formación de las infancias, además de aportar al desarrollo de un profesional integral, comprometido con el medio ambiente y con la transformación de la realidad de las comunidades menos favorecidas, según lo establece la formación por competencias (Aznar, 2003; Fereire, 1997; Gervilla, 2001; Giroux, 1997; Perrenoud, 2004; Porlán y Martín, 1999; Shön, 1992; Tobón, 2007, 2010; Zabalza, 2001).

Ante este escenario, cuestionar y reflexionar constantemente la pertinencia de las propuestas de educación superior diseñadas para la formación de los pedagogos infantiles, constituye una problemática de creciente interés, que además de aportar a una demanda social, favorece la mejora continua de dichos programas, lo cual propende no solo por su sostenibilidad, sino, por la innovación continua de su propuesta de formación.

En este artículo se da cuenta de la experiencia llevada a cabo en la Licenciatura en Pedagogía Infantil de la Corporación Universitaria Minuto de Dios - UNIMINUTO, que al preguntarse sobre ¿cuál es la pertinencia de la propuesta formativa de pedagogos infantiles de UNIMINUTO, respecto a las exigencias actuales de la formación de las infancias? se propone como principal objetivo analizar su propuesta formativa con respecto a las necesidades de formación de la Educación Infantil, con el fin de establecer acciones de mejora y transformaciones del programa, direccionados a generar un aporte de innovación en la educación superior.

El desarrollo de esta iniciativa de investigación está precedido por otros estudios, que aportan en la reflexión.

El primer estudio titulado Percepción del alumnado universitario acerca del uso e integración de las TIC en el proceso educativo de la Facultad de Educación de Granada (Morales, Ortiz, Trujillo y Raso, 2015) evalúa, basado en la percepción de los estudiantes, el uso de las TIC por parte del profesorado universitario, al momento de integrar estas herramientas en su labor educativa. Bajo un paradigma de investigación no experimental, basado en una metodología descriptiva-eclíptica, recoge la información entre los estudiantes a través de cuestionarios y entrevistas.

Una vez triangulada la información, se concluye que en la Facultad de Ciencias de la Educación de la Universidad de Granada, existe una percepción muy favorable respecto a la necesidad de introducir las TIC en la educación superior, pero esto supone una mayor formación por parte del docente universitario, ya que de ello dependerá una mejor integración al acto educativo presencial.

Con estos resultados se pone de manifiesto una fortaleza, pero también un aspecto de mejora por parte de la Facultad de Ciencias de la Educación de la Universidad de Granada, que además de responder ante su compromiso de mejora e innovación permanente, también es resonante ante la necesidad de adaptase a la sociedad actual del conocimiento y la información.

El segundo estudio, también centrado en valorar la formación ofrecida en la educación superior, se titula La evaluación del perfil competencial de alumnos universitarios respecto a la educación tecnológica. Con el objetivo de

conocer y analizar la percepción del alumnado de la Facultad de Ciencias de la Educación de la Universidad de Málaga en relación con la adquisición de las competencias profesionales, referentes a la nuevas tecnologías, necesarias para el desempeño laboral de su profesión" (Díaz y Martín, 2015, p. 17), 
Esta investigación, aplica un cuestionario online tipo likert, bajo una método de investigación descriptiva.

Una vez analizadas las respuestas, se concluye que los estudiantes concuerdan en valorar positivamente el nivel de adquisición de las competencias profesionales, directamente relacionadas con las nuevas tecnologías, siendo estas: la capacidad para manejar de las nuevas tecnologías, la capacidad para aprender nuevos métodos de trabajo, la capacidad para el autoaprendizaje y la capacidad de utilización de diferentes recursos didácticos (pizarras digitales, videos, etc.); sin embargo, también opinan que pese a la favorable percepción sobre el desarrollo de sus competencias, aún les queda mucho por aprender.

Lo cual se constituye en punto de partida para hacer de estos resultados una fuente de información relevante ante la posibilidad de movilizar acciones de mejora en el programa de la Facultad de Ciencias de la Educación de la Universidad de Granada, de acuerdo a las demandas laborales de los futuros profesionales.

En este orden de idea, evaluar la pertinencia de los programas de formación profesional, se posiciona como un eje de trabajo fundamental para las instituciones de educación superior, en la medida que se constituye en la principal herramienta para actualizarse y mantenerse vigente, tal como se constata en el proyecto La Planificación $e$ Innovación de la Evaluación en Educación Superior: la Perspectiva del Profesorado (Quesada, Rodríguez e Ibarra, 2017); de acuerdo al análisis de los resultados de la aplicación de un cuestionario ActEval, a 427 docentes de 18 universidades españolas pone de manifiesto la importancia que le asignan los docentes universitarios al acto de la planificación e innovación. La investigación reconoce que la innovación y el cambio del sistema educativo es una necesidad imperiosa en las instituciones de educación superior para lograr sostenerse y abanderar el "mercado educativo". En este sentido, plantean que la implementación de prácticas educativas mejoradas o innovaciones que perduren en el tiempo, requieren de continuidad y permanencia para garantizar la sostenibilidad necesaria al momento de responder ante las dinámicas de constante cambio educativo, tecnológico y social.

Una vez analizados los anteriores antecedentes, se puede concluir que la evaluación de procesos formativos en la educación superior, para el caso específico de este artículo, en la formación de los futuros docentes de educación infantil, se configura como una necesidad, un reto y compromiso social, que debe responder a las demandas de formación de la infancia, en la medida que garantiza el desarrollo de un profesional integral, comprometido con el medio ambiente, capaz de aportar a la transformación de las realidades menos favorecidas, según lo establece la formación por competencias.

En este sentido, resulta pertinente profundizar en la comprensión conceptual de aquellos elementos que favorecen analizar el nivel de pertinencia de la formación del programa de Pedagogía Infantil de UNIMINUTO, como punto de partida para movilizar transformaciones dirigidas a la innovación en educación superior,. Estas categorías son: formación de pedagogos infantiles, educación infantil, la infancia como una representación social y la evaluación de programa de educación superior

La formación de pedagogos infantiles exige desarrollar competencias generales desde su rol como pedagogo y particulares por su especificidad con la infancia; la integración de estos dos tipos de competencias debe favorecer la consolidación de un ejercicio profesional de carácter democrático, participativo y autónomo, que supere el simple acto discursivo para convertirse en acciones concretas,

Se espera que el pedagogo infantil tenga la capacidad de crear un escenario de aprendizaje, cimentado en un clima de dialogo e interacción horizontal, que medie entre las habilidades, expectativas, sentires, características de desarrollo, diversidad, potencialidades de participación y autonomía del aprendiz, con los objetivos de formación. Todo esto en el marco de la responsabilidad social, en tanto se reconoce como un actor político. Según Freire (1997) el pedagogo infantil debe saber que no se trata solo de enseñar contenidos, sino de dialogar sobre la vida en sí misma; por tanto, se convierte en el "creador de un ambiente abierto y libre dentro del seno de su clase" (p. 97).

El pedagogo infantil debe promover la construcción de saberes, por parte de niños autónomos, logrando así dejar atrás la condición de espectadores pasivos y reproductores de conocimiento elaborados por otros. En este sentido, ejercer la docencia infantil como una práctica política y democrática implica el desarrollo del pensamiento crítico (Sánchez y López, 2016), lo que a su vez, está directamente relacionado con unas competencias comunicativas en un alto nivel de desarrollo (Reche, Martín y Vilches, 2016), todo esto acorde a los retos de la vida profesional en la que deberá ejercer el futuro docente infantil.

Asociado a lo anterior, también se espera que el pedagogo infantil en formación consolide una serie 
de características o más bien atributos que lo perfilan. Freire (1997) habla de cualidades indispensables para el mejor desempeño de los maestros: humildad, amorosidad, valentía, tolerancia, seguridad, sabiduría y alegría.

El pedagogo infantil hasta aquí descrito asume un serio compromiso, ya que en la experiencia de enseñanza- aprendizaje, es el responsable de guiar el proceso del niño al momento de proveer experiencia que les posibilite relacionarse, explorar y conocer el mundo que les rodea; es quien organiza las condiciones y el sistema de influencia educativa para que el infante busque las relaciones esenciales consigo mismo y con los demás, además de construir por sí mismo el conocimiento.

Quiere decir que el papel del pedagogo infantil no es enseñar prematuramente algo que el niño puede descubrir por sí solo, sino proveer experiencias intencionadas a los niños para ir más allá, para que a partir de lo que han hecho o están haciendo, puedan potenciar su desarrollo hasta alcanzar los niveles deseados o sugeridos, según sus características de crecimiento y su entorno sociocultural. Para Zabalza (2001) "El educador debe conocer en cada momento cuál es el nivel de desarrollo de cada niño y para ser capaz de crear un ambiente en el cual se vayan produciendo las actividades esperadas y para apoyarlas cuando éstas ocurran espontáneamente" (p. 215).

Se trata de formar a un pedagogo infantil equilibrado emocional, física, cognitiva y espiritualmente, competente para aportar a la Educación Infantil como un acto complejo y sistémico, un acto vital, multidimensional y singular, diseñado para responder a la pluralidad de la infancia $y$, así mismo, a las necesidades de la realidad actual del conocimiento y la tecnología.

Conceptualizar la Educación Infantil implica reconocer variables como su desarrollo histórico, la nominación que recibe, la edad a la cual está dirigida, los agentes y recursos involucrados, las características de funcionamiento $\mathrm{y}$, de acuerdo a ello, su finalidad e impacto social. Componentes que se articulan de manera dinámica haciendo de dicha definición un fenómeno complejo; sin embargo, el eje central que sustenta su existencia más allá de los matices que pueda asumir dependiendo de la arista desde la cual se defina, es el niño en tanto es reconocido como sujeto con características particulares, necesitado de atención, cuidado y formación por parte de los adultos para suplir exigencias afectivas, biológicas, de protección, participación y desarrollo.

Se desvela la necesidad creciente de ofrecer un servicio equilibrado en el que la formación y el cuidado que se complemente para favorecer al máximo el desarrollo del potencial humano, presente especialmente en la primera etapa de la vida. Sobre este tema, dice Zabalza (1996) "No se trata sólo de que el niño sea feliz y esté cuidado durante esos años. Se trata de hacer justicia a su potencial de desarrollo en unos años que son cruciales" (p. 22).

La educación infantil fundamenta su existencia en el enorme potencial de desarrollo presente en los niños desde el nacimiento. Sobre este concepto Vergara (2008) dice que "el desarrollo se puede identificar como: la integración de conocimientos, de maneras de ser, de sentir, de actuar, que se suscitan al interactuar consigo mismo, con sus padres, con sus pares, con sus docentes, con los objetos" (p. 158).

Reconocidas autoridades de diferentes disciplinas involucradas con la infancia, como el caso de la psicología, la medicina, neurología, historia, nutrición y pedagogía entre otras, acuerdan que los primeros años de vida son una etapa fundamental en el proceso del desarrollo y formación de todos los aspectos que componen al ser humano.

El paradigma de educación infantil desarrollado hasta el momento parte de reconocer la infancia como una representación social que, al estar completamente determinada por el momento histórico y la realidad social actual, se caracteriza por ser plural y transformase constantemente. La infancia se caracteriza por tener diferentes significados que, a su vez, tienen una condición latente a transformarse continuamente, evolucionando de manera histórica.

En este orden de ideas, se puede definir la infancia como una construcción social consensuada y colectivamente compartida. Quiere decir que es aquello que la gente dice o considera que es la infancia en diversos momentos históricos. Cada sociedad, cada cultura, define explícita o implícitamente la infancia, sus características, periodo de vida, implicaciones, conflictos y demás tensiones que posibilitan su existencia. La infancia es una representación que, como fenómeno social, está sujeto a profundas y continuas transformaciones. "Ante la palabra infancia los miembros de un determinado grupo social desarrollan instantáneamente un conjunto de asociaciones mentales y de actitudes compartidas, por la mayoría de los miembros de ese grupo o comunidad" (Rincón et al., 2008, p. 56).

La representación social de la infancia, según Delgadillo (2004) es "como una categoría que se construye histórica, social y culturalmente desde la cotidianidad pero también desde los discursos públicos, académicos y mediáticos" ( $p$ 42). Se 
convierte en el referente que da sentido al comportamiento adulto, al comportamiento de la sociedad en general; de la comprensión que se tenga de infancia dependerán las interacciones sociales, comunicativas y educativas que se establezcan, acerca de o con dicho fenómeno social.

Es claro que la forma en que se ha conceptualizado la infancia $y$, fruto de ello, la manera como ha sido asumida o tratada, tiene una relación directamente proporcional con los componentes educativos vigentes. Los principios de organización educativa y científica dan origen al niño escolar; cuando los principios vigentes son de tipo industrial, se habla de niños trabajadores; si el centro del funcionamiento social está en la familia, surgen los "niños del hogar"; y si el fortalecimiento se encuentra en el Estado, el cuidado adecuado de la infancia está en mano de entidades oficiales creadas para ello (Muñoz y Pacheco, 1996).

Queda evidenciado el impacto directo del proceso socio cultural en la representación social que se gesta de la infancia y su respectiva educación, ya que de la comprensión que de ellos se tiene depende de un conjunto de creencias, valores, lenguaje, objetos, situaciones y expectativas. En este orden de ideas, la representación de infancia de hoy está completamente permeada por la sociedad del conocimiento y la información en la que se destaca la globalización y la complejidad como aspectos que marcan la realidad actual.

Así pues, la educación de la infancia en la sociedad actual se manifiesta bajo una serie de particularidades que la caracterizan y marcan su devenir, pautadas la mayoría de ellas por la prevalecía del conocimiento, la globalización, el constante surgimiento de innovaciones y actualizaciones de las tecnologías de la información y la comunicación, y un creciente posicionamiento de políticas democrática y del cuidado del medio ambiente.

Para De Zubiría (2001) está claro que "vivimos en un mundo profundamente distinto al que conocimos de niños, un mundo en el que la vida económica, política, social, tecnológica y familar es significativamente diferente; responde a otras lógicas, otros espacios, otra realidades y otros tiempo" (p. 13). Realidades a las que definitivamente el pedagogo infantil y en general, el profesional de hoy, está avocado desde su idonediedad profesional.

En este sentido, las propuesta de formación inicial del pedagogo infantil, se ven convocadas a evaluarse constantemente con el propósito de responder a los nuevos retos que plantea la educación de la infancia, que se debe estar renovando constantemente para estar en la capacidad de brindar herramientas para que el futuro docente afronte los cambios y las circunstancias a los que el hecho educativo se ve sometido en la actualidad debido al mundo cada vez más dinámico y globalizado.

Se trata de evaluar sus prácticas educativas para ofrecer una propuesta de formación de pedagogos infantiles, innovadora y comprometida con la creación y transformación acorde a las necesidades cambiantes de la actual sociedad, cada vez más globalizada, informatizada y desafiante Para Marcelo y Vaillant (2009) "Con la formación inicial docente tenemos la oportunidad de crear nuevos docentes apasionados por la enseñanza" (p. 49).

\section{MATERIAL Y MÉTODO}

Para analizar la pertinencia de la propuesta formativa de pedagogos infantil de UNIMINUTO, con respecto a la necesidad de formación de la educación infantil, se presentan parte de los resultados de una investigación de mayor amplitud, titulada "Análisis a la formación de pedagogos infantiles de la Corporación Universitaria Minuto de Dios. Bogotá - Colombia”. Esta investigación llevó a cabo un conjunto de procesos cualitativos y cuantitativos, ya que la articulación de estos enfoques brinda la posibilidad de articular herramientas de recolección de datos acordes con la necesidad investigativa del proyecto en mención.

En coherencia con esta perspectiva, se utilizó un método descriptivo mixto (Hernández, Fernández y Baptista, 2010). Que permite utilizar un conjunto de procesos sistemáticos, empíricos y críticos de la investigación cualitativa y cuantitativa para lograr un mayor entendimiento en el planteamiento del problema, el muestreo, la recolección de datos, el establecimiento de las inferencias y el análisis de los resultados.

Para esta investigación la población fue seleccionada en la Licenciatura en Pedagogía Infantil de la Corporación Universitaria Minuto de Dios -UNIMINUTO de Bogotá - Colombia, que para el 2015 contaba con un total de 1000 estudiantes, 79 graduados, 23 docentes disciplinares, 1 directora de programa, 1 secretaria académica y 1 decano. De acuerdo a esto, la muestra con quien se recolectó la información fue seleccionada a través de un muestreo aleatorio simple; se constituyó por 200 estudiantes y 44 graduados, a quienes se les aplicó una encuesta; 14 docentes, participantes de un grupo focal $\mathrm{y}$, finalmente, una entrevista a cada uno de los 
directivos: directora del programa, secretaría académica y decano de la Facultad de Educación.

Respecto a la encuesta, resulta relevante mencionar que se le hizo la prueba de fiabilidad Alfa de Cronbach, con un resultado de 0,94 tanto para estudiantes como para graduados, lo cual indica una alta fiabilidad del instrumento. Para determinar la validez del mismo se aplicó una prueba piloto y se acudió al juicio de expertos de docentes de la Universidad Santo Tomas y de la Corporación Universitaria Minuto de Dios. Para el proceso de análisis de los datos cuantitativos, la información recibida en las encuestas fue ingresada a una base del programa Microsoft Excel (paquete Microsoft Office XP) y, una vez organizada al información, fue exportada al programa SPSS, versión 21, en donde se hizo el respectivo procesamiento estadístico.

El análisis de los datos cuantitativos se apoyó con la información recolectada en los instrumentos cualitativos: las entrevistas y el grupo focal, información que fue trabajada con el software Atlas Ti, versión 6.2. Una vez procesados los datos cuantitativos y cualitativos, se dio paso a la triangulación entre los datos recolectados, desde una perspectiva teórica, para lograr mayor riqueza, amplitud y profundidad al momento de interpretar la información. Bonilla y Rodríguez (2000) definen la triangulación "como la evaluación de la consistencia de los hallazgos contrastándolos" (p. 152), es decir, que para el caso de esta investigación el contraste se hizo entre los datos cualitativos y cuantitativos obtenidos de los diferentes sujetos de la investigación (estudiantes, graduados, directivos y docentes), estableciendo una relación directa con los referentes conceptuales.

\section{RESULTADOS}

A continuación se presentan los datos cuantitativos y cualitativos más relevantes con respecto al análisis de la pertinencia de la propuesta formativa de pedagogos infantiles de UNIMINUTO, de acuerdo a las necesidades de formación de la infancia como punto de partida para establecer acciones de mejora y transformación del programa. Los resultados cuantitativos se agrupan las respuestas de los estudiantes y los graduados de manera gráfica, con el respectivo análisis. Información que se fortalece con la exposición de los comentarios derivados de los instrumentos cualitativos aplicados a los directivos y a los docentes.

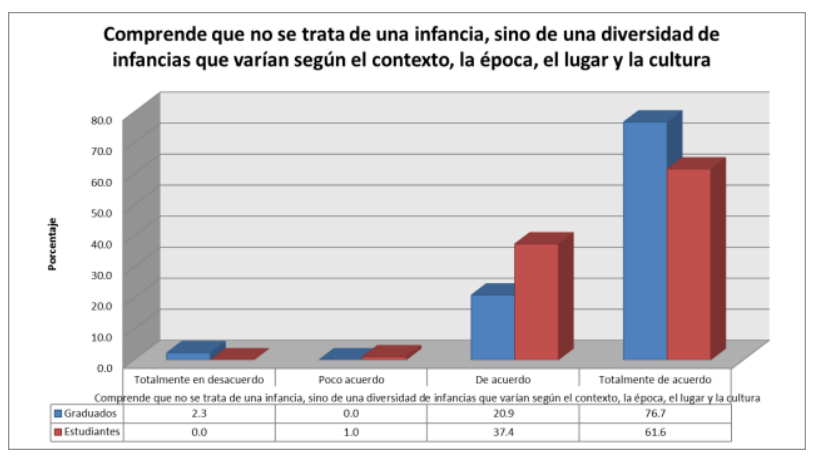

Figura 1. Comprende que no se trata de una infancia, sino de una diversidad de infancias que varían según el contexto, la época, el lugar y la cultura

Definir la infancia más allá de una etapa biológica, como un fenómeno social, implica dejar de lado hablar de una infancia, para darle paso a las infancias, en plural, ya que es posible encontrar tantas infancias como contextos. En este orden de ideas, al interior del programa de Pedagogía Infantil UNIMINUTO, estudiantes en 99\% (De acuerdo y Totalmente de acuerdo) y graduados $97.6 \%$ (De acuerdo y Totalmente de acuerdo) valoran positivamente su capacidad para comprender que no se trata de una infancia, sino de una diversidad de infancias que varían según el contexto, la época, el lugar y la cultura. De los resultados cuantitativos se destaca: "Hablar de infancias en plural, en singular sería un atropello epistémico desde el punto de vista de las culturas entonces" (decano)

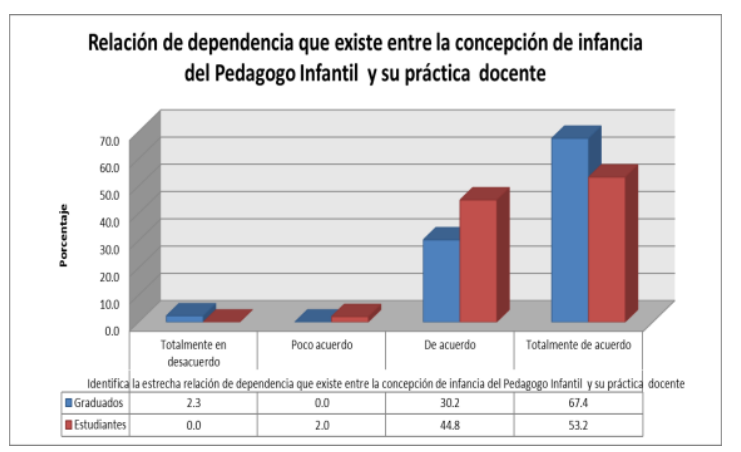

Figura 2. Identifica la estrecha relación de dependencia que existe entre la concepción de infancia del Pedagogo Infantil y su práctica docente

Los estudiantes en 98\% (De acuerdo y Totalmente de acuerdo) y graduados en $97.6 \%$ (De acuerdo y Totalmente de acuerdo) expresan una percepción positiva con respecto a las experiencias de formación ofrecidas por el programa de UNIMINTO, para desarrollar la capacidad de 
identificar la relación de dependencia que existe entre la concepción de infancia del pedagogo infantil y su práctica docente. Quiere decir que el pedagogo infantil UNIMINUTO sabe que el diseño de experiencia de aprendizaje está directamente conectada con su representación social de infancia.

Lo que apoya el decano cuando dice: "La formación en pedagogía infantil tiene que ser desde las pedagogías infantiles desde las infancias por lo tanto una formación desde las infancias implica hablar de pedagogías, de didácticas, de valores en plural".

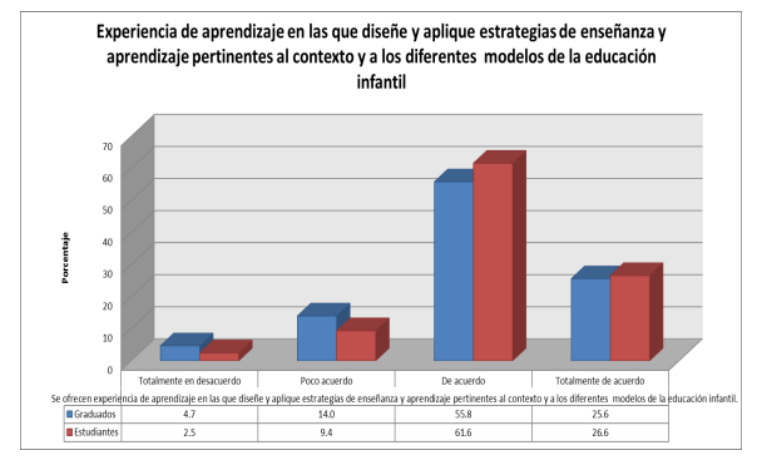

Figura 3. Se ofrecen experiencia de aprendizaje en las que diseñe y aplique estrategias de enseñanza y aprendizaje pertinentes al contexto y a los diferentes modelos de la educación infantil

Coherentes con las respuesta anteriores, estudiantes y graduados concentran las repuestas en De acuerdo, $61.6 \%$ y $55.8 \%$ y en Totalmente de acuerdo en $26.6 \%$ y $25.6 \%$ respectivamente, cuando se les pregunta sobre la experiencia de aprendizaje ofrecida por la Universidad, para desarrollar competencias en el diseño y aplicación de estrategias de enseñanza y aprendizaje pertinentes al contexto y a los diferentes modelos de la educación infantil.

Sobre este aspecto, la secretaría académica expresa: "el programa ha definido las competencias específicas del pedagogo infantil de tal manera que su desempeño en los campos laborales en donde tenga contacto con los niños y niñas con las cuales interactúa sea el mejor profesional"

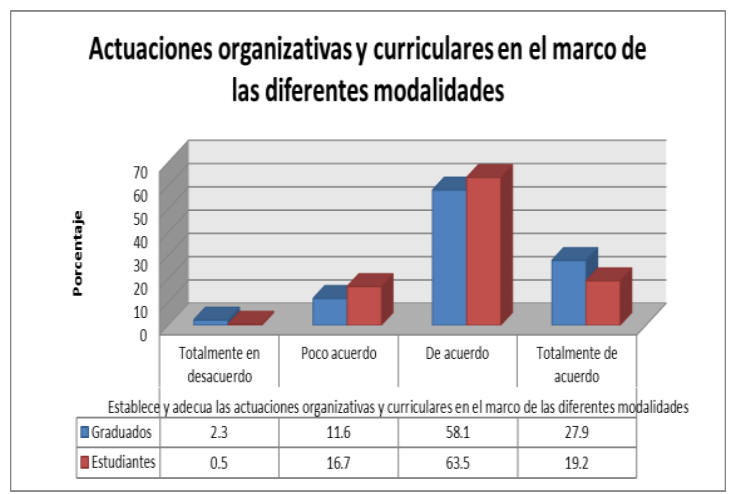

Figura 4. Establece y adecua las actuaciones organizativas y curriculares en el marco de las diferentes modalidades de los centros educativos diseñados para las infancias

Estudiantes con $63,5 \%$ y graduados con $58,1 \%$ coinciden con el mayor porcentaje en De acuerdo, sobre la competencia para diseñar o adaptar, según se requiera, las pautas organizativas y curriculares del acto educativo de las infancias en el marco de las diferentes modalidades de las instituciones comprometidas con la formación de los niños, que para el caso de Colombia son: jardines infantiles, hogares comunitarios, colegios, ONG, centros de desarrollo infantil, ludotecas, aulas hospitalarias y atención a familias bien sea con la modalidad del Instituto Colombiano de Bienestar Familiar (ICBF) o por parte de la Secretaria de Integración Social del Distrito (SDIS).

Por su parte la secretaría académica dice: "podemos revisar los escenarios no solamente son los jardines, no solamente es la escuela, son los parques, son las clínicas, son los museos, en cualquier espacio donde hay una familia, en las mismas iglesias, en fin hay posibilidades realmente muy amplias. No se limite al aula sino que realmente se expanda a las juntas de acción comunal, las parroquias, todos esos espacios hay una oportunidad súper valiosísima para el pedagogo infantil poder tener contacto con los niños, donde hay un niño ahí debe estar un maestro de pedagogía infantil". 


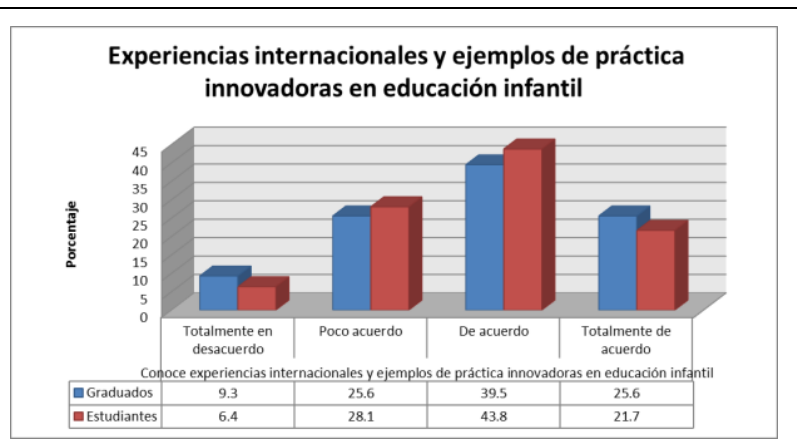

Figura 5. Conoce experiencias internacionales y ejemplos de práctica innovadoras en educación infantil

Las competencias sobre los diferentes escenarios y modalidades de la educación infantil en Colombia, se complementa con el conocimiento que graduados $\mathrm{y}$ estudiantes dicen tener sobre las experiencias y ejemplos de prácticas innovadores en educación infantil a nivel internacional. Con un total de $65,1 \%$ entre De acuerdo y Totalmente de acuerdo por parte de los graduados y $65,5 \%$ sumando De acuerdo y Totalmente de acuerdo de los estudiantes, ambos grupos de encuestados manifiestan una percepción positiva.

En palabras de la directora del programa "se brindan oportunidades de aprendizaje en la que identifican la educación infantil en otros países, un ejemplo de ello son las experiencia de movilidad internacional gracias a la cuales diferente estudiantes han tenido la oportunidad de hacer cursos de corta duración en Cuba y en Brasil, además de los intercambio de semestres que se han llevado a cabo en Argentina y España, por parte de diferentes estudiantes"

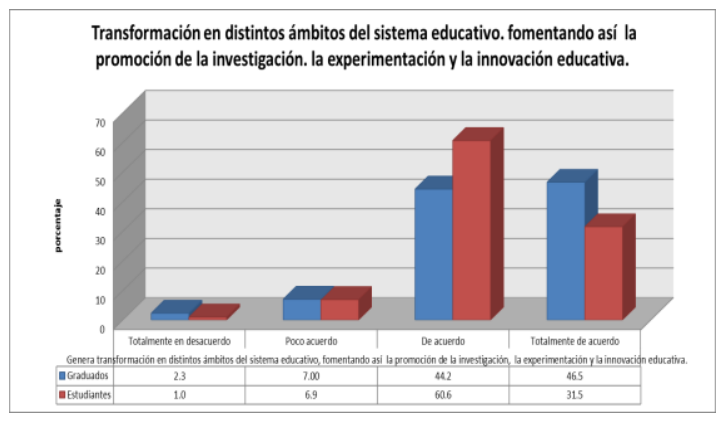

Figura 6. Genera transformación en distintos ámbitos del sistema educativo, fomentando así la promoción de la investigación, la experimentación y la innovación educativa.

Sobre este aspecto, la respuesta de los estudiantes y los graduados nuevamente se agrupa en un alto nivel, en De acuerdo y Totalmente de acuerdo, $92.1 \%$ y $90.7 \%$, respectivamente. Desde esta perspectiva, se puede inferir que la propuesta de formación de pedagogos infantiles UNIMINUTO, desarrolla capacidades en sus estudiantes para generar transformaciones en distintos ámbitos del sistema educativo, fomentando así la promoción de la investigación, la experimentación y la innovación educativa.

Al respecto, la coordinadora del departamento indica: "Generar unas actitudes de criticidad y de reflexión de nuestra propia práctica y ese postulado que uno sea capaz de mirarse de ser crítico frente uno mismo y que quiera generar cambios inmediatamente lo pone uno en una posición en un lugar innovado".

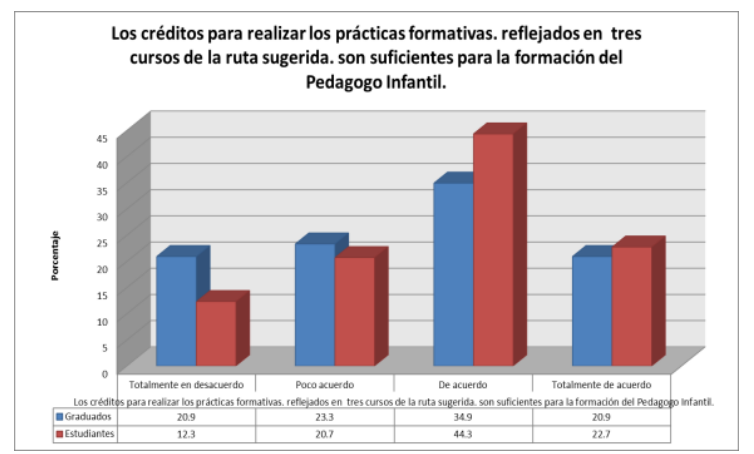

Figura 7. Los créditos para realizar los prácticas formativas, reflejados en tres cursos de la ruta sugerida, son suficientes para la formación del Pedagogo Infantil.

Aunque en esta pregunta también prevalece una percepción positiva, es la primera, en la que suben significativamente las respuestas en Poco de acuerdo y Totalmente de desacuerdo, $33.0 \%$, estudiantes y $44.2 \%$, graduados, contra un $67 \%$ y $55.8 \%$, a favor, respectivamente. Este incremento de los porcentajes, permite inferir que para las dos poblaciones, las tres prácticas, las cuales solo dos son profesionales, resultan insuficientes para su formación como pedagogos infantiles.

Resultado que se articula con la siguiente afirmación de un docente: "yo creo que a nivel de prácticas, hay que seguir potenciando eso porque es que lo estamos viendo tal vez en los espacios académicos yo veo que todas hacemos ejercicios muy interesantes y muy prácticos para que pase eso, pero a nivel ya al final en el ejercicio de la práctica de pronto algo está pasando porque estamos perdiendo eso y la estudiante se está encasillando en los sistemas que ya están ahî" (docente 2). 


\section{DISCUSIÓN}

En respuesta a la creciente necesidad de reflexionar, analizar y mejorar la propuesta de formación de pedagogos infantiles de UNIMINUTO, como punto de partida para aportar a la innovación en educación superior, resulta relevante destacar que:

El diseño curricular reflejado en el plan de estudios de la licenciatura de Pedagogía Infantil ofrece experiencias de aprendizaje en las que el pedagogo infantil reconoce las infancias como un grupo poblacional con derechos específicos; por tanto, les brindan el estatus de ciudadano de acuerdo a las características específicas y diversidad multicultural, lo que va ligado a entender que las infancias son una representación social directamente relacionada con la diversidad social, cultural e histórica. Desde esta perspectiva, el pedagogo infantil UNIMINUTO, desarrolla la competencia para diseñar y aplicar estrategias de enseñanza y aprendizaje basadas en la diversidad y pluralidad infantil, lo cual las hace pertinentes al contexto y a los diferentes modelos de la educación infantil.

Aspecto sobre el cual también profundizan y concuerdan Peña y Durán (2011), en su investigación La Triada Social por la Infancia, específicamente en la unidad de análisis sobre formación docente, desde donde concluyen que la Licenciatura en Pedagogía Infantil de la Universidad de Pamplona ofrece a sus estudiantes una serie de espacios formativos que permiten "la consolidación de un profesional en educación competente para comprender el desarrollo infantil y a partir de ello, proponer experiencias de aprendizaje en los espacios escolares y no escolares" (Peña y Durán, 2015 p. 100).

Respecto a las modalidades de la educación infantil, los escenarios a los cuales el pedagogo UNIMINUTO tiene mayor acceso durante su proceso de formación son: colegios oficiales y privados, hogares comunitarios, jardines infantiles y ONG. Esto quiere decir que el pedagogo infantil de UNIMINUTO es idóneo ante los requerimientos académicos - administrativos de centros educativos como: jardines infantiles, hogares comunitarios, colegios, ONG, centros de desarrollo infantil, ludotecas, aulas hospitalarias y atención a familias bien sea con la modalidad del Instituto Colombiano de Bienestar Familiar (ICBF) o por parte de la Secretaria de Integración Social del Distrito (SDIS).

Sin embargo, el programa se plantea como reto de mejora, ampliar mucho más esta gama de escenarios en busca de consolidar una mayor diversificación que responda por la formación de la infancia ubicada en los hospitales, hogares infantiles nocturnos, museos y centros comerciales entre otros. También surge el reto de transformar los procesos de formación relacionados con las prácticas profesionales, ya que los créditos para realizar los prácticas formativas, reflejados en tres cursos de la ruta sugerida, son insuficientes para la formación del pedagogo infantil.

Para afrontar este reto de mejora se propone una nueva configuración del proceso de prácticas, que en relación directa con el modelo educativo de UNIMINUTO, basado en la praxeología, tendrán una duración de 50 créditos, con una complejidad creciente, iniciando con la observación hasta terminar con la intervención pedagógica. Lo que resulta completamente compatible y congruente con los aportes que conlleva el enfoque praxeológico en la formación de pedagogos infantiles (Carvajal, 2016) y la necesidad de diseñar estrategias pedagógicas que, basadas en el desarrollo del pensamiento crítico, se configuren como un aporte de innovación educativa en la educación superior latinoamericana (Aznar y Laiton, 2017).

$\mathrm{La}$ licenciatura en Pedagogía Infantil UNIMINUTO asume el llamado a la investigación, la experimentación y la innovación educativa como un principio que le permite analizar, transformar y mejorar continuamente su propuesta de formación de docentes, para con ello responder a las cambiantes demandas educativas de las infancias. Esta perspectiva que impulsa la innovación en educción superior es ratificada por Rodríguez., González y Gámiz (2016) en su análisis sobre la líneas y experiencias de innovación en educación superior de la Universidad de Murcia y la Universidad de Granada, implementadas con el propósito de responder con mejores índices de eficacia y eficiencia la enseñanza y el aprendizaje del estudiante universitario.

\section{CONCLUSIONES}

El análisis de la pertinencia de la propuesta formativa de pedagogos infantiles de UNIMINUTO, de acuerdo a las necesidades de formación la infancia como punto de partida para establecer acciones de mejora y transformación del programa, direccionados a generar un aporte de innovación en la educación superior, permite concluir que:

La propuesta de formación de pedagogos infantiles UNIMINUTO favorece que el futuro docente consolide un pensamiento crítico, comprometido con la transformación de las realidades educativas de los niños, en especial de aquellos menos favorecidos, dada su capacidad de 
hacer una lectura analítica, reflexiva y argumentada del contexto, como punto para intervenir el contexto con una propuesta pedagógica basada en el reconocimiento de la singularidad, pluralidad y diversidad de las infancias, las cuales constantemente demandan nuevos retos de formación.

El pedagogo Infantil UNIMINUTO tiene la capacidad de reconocer al niño como un sujeto de derecho que se caracteriza por ser social, único y singular. Este aspecto se constituye en la base al momento de promover experiencias de aprendizaje incluyentes, centradas en el desarrollo de las potencialidades de cada estudiante, de acuerdo a sus intereses y necesidades particulares. .

El principal necesidad de mejora es la ampliación y diversificación la práctica formativa. Al respecto se proponen dos retos: a) Fortalecer el plan de estudios, incrementado de trece créditos, a cincuenta créditos de práctica in situ, con los respectivos cursos o seminarios de apoyo y b) Diversificar los escenarios de práctica mediante la creación de convenios con aula hospitalarias, hogares infantiles nocturnos, práctica rurales, ludotecas, museos, entre otros, en general en todos los escenarios no convencionales en los que también resulta fundamental formar a la infancia.

\section{REFERENCIAS}

Alzate, M. V. (2002). Concepciones e imágenes de la infancia. Revista de Ciencias Humanas. 28, 1-13. Recuperado de http://repository.unad.edu.co/bitstream/10596/4863/1/51 4517\%20infancia.pdf

Alzate, M. V. (2003). Las infancias: concepciones $y$ perspectivas. Pereira: Papiro

Amar, J., Abello, R. y Tirado, D. (2014). Desarrollo Infantil y Construcción del mundo Social. Barranquilla: Editorial Universidad del Norte.

Arnaiz, P. (2003). Educación Inclusiva: una escuela para todos. Málaga: Ediciones Aljibe.

Aznar Díaz, I. (2003). Metodología Didáctica, Acción Docente y Desarrollo del alumnado de Educación Infantil y Primaria. Granada: ReproDigital.

Aznar, Díaz, I y Laiton, I (2017). Desarrollo de Habilidades Básicas de Pensamiento Crítico en el Contexto de la Enseñanza de la Física Universitaria. Formación Universitaria Vol. 10(1), 71-78. DOI: 10.4067/S071850062017000100008

Bonilla, E. y Rodríguez, P. (2000). Más allá del dilema de los métodos. Bogotá: Norma

Casado, E. (2001) La teoría de las representaciones sociales. CINDE Conocimiento Social y socialiación y Política. Venezuela: Escuela de Educación Universidad Central de Venezuela

Casas, F. (1998). Infancia: perspectivas psicosociales. Barcelona: Paidós.

Casas, F. (2006). Infancia y representaciones sociales. Política y Sociedad, 48(1), 27-42. Recuperado en
http://revistas.ucm.es/index.php/POSO/article/view/POS O0606130027A/22636

Castells, M. (1999). La Era de la información: Economía, sociedad y cultura. (Vol 1). México: Siglo XXI.

Carvajal, M. (2016). La pedagogía praxeológica como componente en el proceso de investigación para la formación ciudadana. Educ. Educ., 19(3), 416-436. DOI: 10.5294/edu.2016.19.3.6

De Zubiría, J. (2011). Los modelos pedagógicos: Hacia una pedagogía dialogante. Bogotá : Magisterio

Delgadillo, I. (2004). La infancia en la perspectiva de las representaciones sociales. Pedagogía y Saberes, 20, 4152.

Díaz, M., y Martín Jaime, J. (2015). Evaluación del perfil competencial de alumnos universitarios respecto a la educación tecnológica. Innoeduca. International Journal of Technology and Educational Innovation, 1(1), 17-24. doi:http://dx.doi.org/10.20548/innoeduca.2015.v1i1.29

Diker, G. (2009). ¿Qué hay de nuevo en las infancias?. Buenos Aires: Universidad Nacional de General Sarmiento y Biblioteca Nacional

Freire, P. (1997). Cartas a quien pretende Enseñar. México: Siglo XXI.

Gervilla, Á. (2001). Didáctica y formación del profesarado ¿Hacia un nuevo paradigma?. Madrid: Dykinson.

Gervilla, Á. (2002). Necesidades Educativa de la Infancia ante el nuevo milenio. Madrid: CEDMA.

Gervilla, Á. (2006). Didáctica básica de la educación infantil. Conocer y comprender a los más pequeños. Madrid: Narcea.

Gervilla, Á. (2006). El currículo de la Educación Infantil. Madrid: Narcea.

Giroux, H. (1997). Los profesores como intelectuales. Madrid: Paidós.

Hernández, R., Fernández, C. y Baptista, M. (2010). Metodología de la Investigación. México: McGraw-Hill.

Jodelet, D. (2007). Imbrincaciones entre representaciones sociales e intervención T. Rodríguez y M. de L. García (Ed.). Guadalajara: Universidad de Guadalajara.

Marcelo, C. y Vaillant, D. (2009). Desarrollo Profesoral Docente. ¿Cómo se aprende a enseñar? Madrid: Narcea.

Mejía, R. (2006). Educación (es) en la (s) globalización (es) I. Bogotá: Ediciones desde abajo

Morales Capilla, M., Ortíz Colón, A., Trujillo-Torres, J., y Raso Sánchez, F. (2015). Percepción del alumnado universitario acerca del uso e integración de las TIC en el proceso educativo de la Facultad de Educación de Granada. Innoeduca. International Journal of Technology and Educational Innovation, 1(2), 57-68. doi:http://dx.doi.org/10.20548/innoeduca.2015.vli2.103 5

Moscovici, S. (1985). Psicología Social, I. Barcelona: Paidós.

Muñoz, C. y Pacheco, P. (1996). La aventura infantil a mediados de siglo. Bogotá: Multiletras

Nussbaum, M. (2012). Crear capacidades para el desarrollo humano. Bogotá: Paidos

Peña, C. y Durán, Y. (2011). La formación de Educadores Infantiles en la Universidad de Pamplona: Análisis de una experiencia significativa. Revista Educación y Ciudad. 20, 99-116.

Peralta, M.V. (2003). Los desafíos de la educación Infantil en el Siglo XXI y sus implicaciones en la formación y prácticas de los agentes educativos. Chile: Ministerio de Educación.

Perrenoud, P. 2004. Diez nuevas competencias para enseñar. Invitación al viaje. Barcelona : Grao. 
Porlán, R. y Martín, R. (1999). Tendencias en la formación incial del profesorado sobre los contenidos escolares. Rev. Interuniv. From. Profr, 35, 115 - 128.

Quesada Serra, V., Rodríguez Gómez, G. e Ibarra Sáiz, M.S. (2017). Planificación e innovación de la evaluación en educación superior: la perspectiva del profesorado. Revista de Investigación Educativa, 35(1), 53-70. DOI: http://dx.doi.org/10.6018/rie.35.1.23926153

Reche Urbano, E., Martín Fernández, M., y Vilches Vilela, M. (2016). La competencia literaria y comunicativa en la formación inicial del docente. Presentación de una experiencia. Innoeduca. International Journal of Technology and Educational Innovation, 2(2), 138-144. doi:http://dx.doi.org/10.20548/innoeduca.2016.v2i2.203 5

Rey, G. (2002). Cultura y desarrollo humano: Unas relaciones que se trasladan en Pensar Iberoaméricana: Revista de cultura. $\quad \mathrm{N}^{\circ}$ 0, Bogotá, OEI. Recuperado en http://www.oei.es/historico/pensariberoamerica/ric00a04 .htm

Rincón, C. et al. (2008). Imaginarios de infancia y la formación de maestros. Bogotá: Alcaldía Mayor de Bogotá, Instituto para la Investigación Educativa y el Desarrollo Pedagógico - IDEP, Colciencias y Universidad Distrital Francisco José de Caldas.

Rodríguez-Entrena, M.J., González-Barea, E.M y Gámiz, V. (2016). La perspectiva de innovación que se impulsa desde la Educación Superior. Journal for Educators, Teachers and Trainers, Vol. 7(1). 193 - 209. Recuperado http://jett.labosfor.com/index.php/jett/article/view/199/2 49

Sánchez Uceda, M., y López Regalado, O. (2016). El CmapTools en estudiantes universitarios para desarrollar pensamiento crítico. Innoeduca. International Journal of Technology and Educational Innovation, 2(1), 54-63. doi:http://dx.doi.org/10.20548/innoeduca.2016.v2i1.103 7

Schön, D. (1992). La formación de profesionales reflexivos. Barcelona: Paidos

Sen, A. (2000). Desarrollo y Libertad: Buenos Aires: Editorial Planeta

Tobón, S. (2007). Competencias en Educación Superior: Políticas hacia la Calidad. Bogotá: Ecoe Ediciones.

Tobón, S. (2010). Formación integral y competencias. Pensamiento complejo, currículo, didáctica y evaluación. Bogotá: Ecoe

Trujillo, M. y Martín, S. (2010). Desarrollo Socioafectivo. Madrid: Editorial Editex

Vergara, V. (2008). La Educación Inicial. Guía de gestión, fundamentación y aplicación. Bogotá: Andeip.

Zabalza, M. Á. (1996). Calidad en la Educación Infantil. Madrid: Narcea.

Zabalza, M. Á. (2001). La Autoestima de los Educadores. Congreso Europeo: Aprender a ser, aprender a vivir juntos.

Zabalza, M. Á. (2008). Didáctica de la educación infantil. Madrid: Narcea 